\title{
Fortaleciendo capacidades para la innovación - Un modelo para reducir la pobreza usando papas nativas
}

\author{
J. Andrade-Piedra ${ }^{1}$, A. Thomann ${ }^{2}$, C. Velasco ${ }^{3}$, C. Pérez ${ }^{1}$, E. Quino ${ }^{4}$, N. Cussy ${ }^{4}$, H. \\ Foronda $^{4}$, E. Gandarillas ${ }^{5}$, R. Esprella ${ }^{5}$, P. Flores ${ }^{5}$, C. Falconí ${ }^{6}$, R. Garzón ${ }^{6}$, Á. Salinas $^{6}$, J. \\ López $^{6}$, M. Quispillo ${ }^{6}$, I. Reinoso ${ }^{7}$, F. Yumisaca ${ }^{7}$, P. Llangarí ${ }^{7}$, F. Haro ${ }^{7}$, M. Cuentas ${ }^{8}$, P.

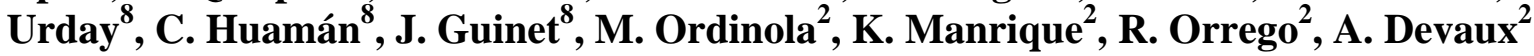

\section{RESUMEN}

Este artículo describe de manera preliminar un modelo de intervención que tiene como objetivos: (i) complementar al Enfoque Participativo de Cadenas Productivas (EPCP) y las Plataformas de Concertación con acciones para fortalecer las capacidades de organizaciones de agricultores de bajos recursos para generar y aprovechar innovaciones y, como resultado, mejorar los ingresos y bienestar de los agricultores; y (ii) mejorar la conexión de la investigación con el desarrollo probando tecnologías y metodologías desarrolladas por el Centro Internacional de la Papa (CIP) y sus socios, generando bienes públicos internacionales. Al aplicar el modelo en Bolivia, Ecuador y Perú, se incrementaron los rendimientos y calidad de papas nativas, y varias organizaciones de agricultores accedieron a mercados de alto valor, resultando en un incremento significativo en el ingreso de 200 familias en Bolivia, 255 en Ecuador y 131 en Perú. También se creó capital social entre las instituciones que implementaron el modelo y se retroalimentó su agenda de investigación con temas demandados por el mercado y los agricultores. Este modelo de intervención puede convertirse en un bien público para el CIP y otras instituciones de investigación y desarrollo, ya que puede mejorar los efectos e impactos de sus tecnologías y metodologías. Sin embargo, es necesario más tiempo y análisis para desarrollar el modelo y evaluar su validez.

Palabras Clave: biodiversidad, acceso a mercado.

\section{Strengthening capacities for innovation - A model for reducing poverty using native potatoes}

\author{
ABSTRACT

\footnotetext{
${ }^{1}$ Centro Internacional de la Papa (CIP), Quito, Ecuador. j.andrade@cgiar.org

${ }^{2}$ CIP, Lima, Perú.

${ }^{3}$ CIP, Cochabamba, Bolivia.

${ }^{4}$ Asociación KURMI, La Paz, Bolivia.

${ }^{5}$ Fundación PROINPA, La Paz, Cochabamba, Bolivia.

${ }^{6}$ Fundación M.A.R.CO., Riobamba, Ecuador.

${ }^{7}$ INIAP, Quito, Riobamba, Ecuador.

${ }^{8}$ Asociación FOVIDA, Lima, Perú.
}

This paper describes preliminarily an intervention model with the following objectives: (i) to complement the Participatory Market Chain Approach (PMCA) and the Multi-Stakeholder Platforms with actions for strengthening the capacities of low-resource farmers' organizations to generate and use innovations and, as result, improve the income and wellbeing of farmers; and (ii) improve the connection between research and development, testing technologies and methodologies developed by the International Potato Center (CIP) and its partners, generating international public goods. The model was applied in Bolivia, Ecuador 
Andrade-Piedra, Thomann, Velasco, Pérez, Quino, Cussy, Foronda, Gandarillas, Esprella, Flores, Falconí, Garzón, Salinas, López, Quispillo, Reinoso, Yumisaca, Llangarí, Haro, Cuentas, Urday, Huamán, Guinet, Ordinola, Manrique, Orrego, Devaux

and Peru. As result potato yield and quality were improved, and several farmers' organizations accessed to high value markets, resulting in a significant income increase for 200 families in Bolivia, 255 in Ecuador and 131 in Peru. Social capital was created among institutions that implemented the model and their research agendas were feedbacked with subjects demanded by the market and farmers. This intervention model can become a public good for CIP and other research and development organizations, because it can improve the outcomes and impacts of their technologies and methodologies. However, more time and analysis is needed to develop the model and evaluate its validity.

Key words: biodiversity, market access.

\section{Introducción}

En los Andes de Bolivia, Ecuador y Perú se concentra la mayor diversidad de papa a nivel mundial y al mismo tiempo se encuentran altos niveles de pobreza. Una estrategia para reducir dicha pobreza consiste en conectar a los agricultores de bajos recursos a mercados de alto valor que reconozcan las bondades culturales, alimenticias y ambientales de las papas nativas. Para ello, conceptos de acción colectiva (Johnson y Berdegué, 2004) y de sistemas de innovación (World Bank, 2007) han sido utilizados por la Iniciativa Papa Andina del Centro Internacional de la Papa (CIP) y sus socios para desarrollar enfoques como el Enfoque Participativo de Cadenas Productivas (Bernet et al., 2006) y las Plataformas de Concertación (Devaux et al., 2009). Primeros resultados de la aplicación de estos enfoques muestran que son válidos para mejorar los ingresos de agricultores de bajos recursos (e.g., Cavatassi et al., 2009).

Lo anterior ocurre en un contexto en el que el CIP y otros centros de investigación mundial tienen el desafío de contribuir a las Metas de Desarrollo del Milenio. Para cumplir este desafío es indispensable mejorar la conexión de la investigación con el desarrollo.

Este artículo describe un modelo de intervención que tiene como objetivos: (i) complementar al EPCP y las Plataformas con acciones para fortalecer las capacidades de organizaciones de agricultores de bajos recursos para generar y aprovechar innovaciones $\mathrm{y}$, como resultado, mejorar los ingresos y bienestar de los agricultores; y (ii) mejorar la conexión de la investigación con el desarrollo probando tecnologías y metodologías desarrolladas por el CIP y sus socios, generando bienes públicos internacionales.

\section{Materiales y métodos}

El modelo se desarrolló en base a trabajo de campo iniciado a mediados del 2006. En Bolivia se trabajó en 11 comunidades de La Paz con papas nativas para producción de papa deshidratada en frío (chuño); en Ecuador en 7 comunidades de Chimborazo con papas mejoradas y nativas para mercado en fresco y leche para producción de queso y yogur; y en Perú en 3 comunidades de Junín y Huancavelica con papas nativas para producción de hojuelas. En coordinación con CIP, dos instituciones en cada país - Fundación PROINPA y Asociación Kürmi en Bolivia, INIAP y Fundación MARCO en Ecuador, y proyecto INCOPA y Asociación FOVIDA en Perú - implementaron acciones en 5 ejes temáticos en base a una teoría de cambio que vinculó el fortalecimiento de capacidades para generar innovaciones con el mejoramiento de ingresos de los agricultores.

En el primer eje temático (acceso a mercado) se entrenó a líderes agricultores en el manejo de herramientas de gestión y se facilitó el contacto con empresas transformadoras y comercializadoras. En el segundo eje (acceso a información) se desarrollaron sistemas de información comercial y tecnológica. En el tercer eje (acceso a tecnologías de producción) se capacitó a productores mediante Escuelas de Campo de Agricultores y Comités de Investigación Agrícola Local, y se desarrollaron investigaciones en base a demandas de mercado. 
En el cuarto eje (intercambio de experiencias), se realizaron sistematizaciones y visitas de campo a nivel nacional, y varias Evaluaciones Horizontales (EH) (Thiele et al., 2007) a nivel regional. Finalmente, en el quinto eje (género) se diseñó e implementó una estrategia para asegurar que hombres y mujeres puedan acceder a las intervenciones arriba descritas.

\section{Resultados}

De manera preliminar se determinó que se incrementaron los rendimientos y calidad de papas nativas y leche (este último rubro en Ecuador). Además, las organizaciones de agricultores accedieron a mercados de alto valor a través de la empresa Fritolay en Perú, de la plataforma AndiBol en Bolivia, y de las organizaciones de agricultores CONPAPA y La Quesera en Ecuador. Existió un incremento significativo en el ingreso de 200 familias en Bolivia, 255 en Ecuador y 131 en Perú.

También se creó capital social entre las instituciones socias. Este capital social se expresó como una mayor confianza y una mejor actitud para generar innovaciones comerciales, tecnológicas e institucionales.

Finalmente, se retro-alimentó la agenda de investigación de CIP, PROINPA e INIAP con temas demandados por el mercado y los agricultores (e.g., fertilización para mejorar la calidad de fritura, manejo poscosecha de chuño, selección de clones para mercado en fresco) y se generó una guía para facilitación de EH para ser usada en otros contextos como bien público.

\section{Discusión}

El modelo de intervención aquí descrito consiste en fortalecer capacidades de organizaciones de agricultores para que aprovechen la biodiversidad de papa y otros productos de su sistema de producción y que mejoren sus relaciones con otros actores de la cadena productiva, de manera que pueda usar y participar en la generación de innovaciones tecnológicas, comerciales e institucionales. Con esto se busca que se conecten a mercados de alto valor y así mejoren sus ingresos y bienestar. Esto lo hace a través de socios estratégicos (instituciones de investigación nacionales) y socios locales (ONGs de desarrollo) en territorios específicos, bajo la coordinación y el liderazgo de un centro de investigación internacional (CIP). Este último pone a disposición metodologías, tecnologías y capacidad de investigación y busca que el modelo sea replicable en otros contextos.

Este modelo permite complementar al EPCP y a las Plataformas de Concertación. En ambas metodologías la participación de las organizaciones de agricultores ha sido relegada a un segundo plano pero ahora, con capacidades fortalecidas, pueden asumir un rol protagónico en negociaciones con otros actores de la cadena. Además, pueden ser parte de las acciones de incidencia pública y política que realizan la Iniciativa Papa Andina y sus socios. De esta manera, este modelo de intervención puede servir para mejorar la conexión entre los niveles locales (comunidad) y los niveles regionales (departamento o provincia) y nacionales.

Aunque se mencionan varios temas que han sido incorporados en las agendas de investigación de los socios y se menciona un bien público que ha sido generado a través de la implementación del modelo, aún falta camino por recorrer para mejorar la conexión entre investigación y desarrollo. Por ejemplo, se subestimó el tiempo que lleva construir una agenda común entre investigadores y técnicos de desarrollo. También es necesario realizar evaluaciones detalladas de efectos e impactos para asegurar que el modelo de intervención sea válido.

\section{Conclusión}

Este modelo de intervención puede convertirse en un bien público para el CIP y otras instituciones de investigación y desarrollo, ya que puede mejorar los efectos e impactos de sus tecnologías (e.g., manejo integrado de plagas) y metodologías (e.g., EPCP). Sin embargo, es necesario más tiempo y análisis para desarrollar el modelo y evaluar su validez. 
Andrade-Piedra, Thomann, Velasco, Pérez, Quino, Cussy, Foronda, Gandarillas, Esprella, Flores, Falconí, Garzón, Salinas, López, Quispillo, Reinoso, Yumisaca, Llangarí, Haro, Cuentas, Urday, Huamán, Guinet, Ordinola,

\section{Manrique, Orrego, Devaux}

\section{Agradecimientos}

Este trabajo se realizó con fondos de la Agencia de Desarrollo y Ayuda Internacional de Nueva Zelandia (NZAid) a través del proyecto "Fortalecimiento de las capacidades para innovar y reducir la pobreza en los Andes, InnovAndes”.

\section{Literatura citada}

Bernet, T., Thiele, G., Zschocke, T. 2006. Participatory Market Chain Approach (PMCA) User Guide. International Potato Center (CIP), Lima - Perú. 169 p.

Cavatassi, R., Gonzalez, M., Winters, P., Andrade-Piedra, J.L., Espinosa, P. and Thiele, G. 2009. Linking Smallholders to the New Agricultural Economy: An Evaluation of the Plataformas Program in Ecuador. Agricultural Development Economics Division (ESA), Food and Agriculture Organization (FAO). Working Paper No. 09-03.
Devaux, A., Douglas, H., Velasco, C., Thiele, G.,López, G., Bernet, T., Reinoso, I., Ordinola, M. 2009. Collective action for market chain innovation in the Andes. Food Policy 34(1):3138.

Johnson, N., Berdegué, J. 2004. Property rights, collective action and agribusiness. Brief for "Collective Action and Property Rights for Sustainable Development”, 2020 Vision for Food, Agriculture and the Environment. Focus 11, Brief 13. IFPRI, Washington, DC.

Thiele, T., Devaux, A., Velasco, C., Horton, D. 2007. Horizontal evaluation - Fostering knowledge sharing and program improvement within a network. American Journal of Evaluation 28 (4): 493-508.

World Bank. 2007. Enhancing agricultural innovation: how to go beyond the strengthening of research systems. Washington, D.C.: The World Bank. Agric. and Rural. Devel. Depart. 